\title{
Fixed points for four maps related to generalized weakly contractive condition in partial metric spaces
}

\author{
M. Bousselsal ${ }^{1 *}$, M.S. Jazmati ${ }^{2}$ \\ ${ }^{1}$ Laboratoire d'Analyse Nonlineaire, Dept. of Maths ENS-Kouba, 16050, Vieux-Kouba Algiers ( Algeria) \\ 2 Dept. of Maths. College of Science, Qassim University P.O. Box: 6644, Bouraida, 51452, Saudi Arabia (KSA) \\ *Corresponding author Email: bousselsal55@gmail.com
}

Copyright (C)2015 M. Bousselsal, M.S. Jazmati. This is an open access article distributed under the Creative Commons Attribution License, which permits unrestricted use, distribution, and reproduction in any medium, provided the original work is properly cited.

\begin{abstract}
We prove a unique fixed point theorem for a function depending from four self maps satisfying $(\phi-\psi)$-contractive condition in partial metric spaces. Presented results extend and generalize some existing fixed point results in the literature.
\end{abstract}

Keywords: Partial metric; Weakly compatible maps; Complete space.

\section{Introduction}

The Notion of partial metric space have originally developed by S.G. Matthews ([3]) to provide mechanism generalizing metric space theories. This relatively new field has been shown to have vast application potentials [6] in the study of computer domains and semantics [7]. The partial metric spaces play an important role in constructing models in the theory of computation see $[1,3,6,8]$.

S.G Matthews ([3])., Sandra Oltra and Oscar Valero [8], Salvador Romaguerra [9], I. Altun, Ferhan Sola [1] and K.P.R Rao and G.N.V. Kishore [5] proved fixed point theorems in partial metric spaces for a single map.

In this paper, we prove a unique fixed point theorem for four self mappings for a generalized operator depending from $(\psi-\varphi)$ contractive condition in partial metric spaces.

First, let us recall some definitions and lemmas of partial metric spaces that we will use in the sequel.

\section{Preliminaries}

Definition 2.1 (([3]).). A partial metric on a nonempty set $X$ is a function $p: X \times X \rightarrow R^{+}$such that for all $x, y, z \in X:$

$\left(p_{1}\right) x=y \Longleftrightarrow p(x, x)=p(x, y)=p(y, y)$,

$\left(p_{2}\right) p(x, x) \leq p(x, y), p(y, y) \leq p(x, y)$,

$\left(p_{3}\right) p(x, y)=p(y, x)$,

$\left(p_{4}\right) p(x, y) \leq p(x, z)+p(z, y)-p(z, z)$.

A partial metric space $(X, p)$ is a pair $(X, p)$ such that $X$ is a nonempty set and $p$ is a partial metric on $X$. 
Remark 2.2 (([3]).). It is clair that

a) $|p(x, y)-p(y, z)| \leq p(x, z), \forall x, y, z \in X$.

b) $p(x, y)=0 \Longrightarrow x=y$.

c) If $x=y, p(x, y)$ may not be zero. We consider the following counter-example, the pair $\left(R^{+}, p\right)$, where $p(x, y)=$ $\max \{x, y\}$ for all $x, y \in R^{+}$.

d) If $p$ is a partial metric on $X$, then the function $p^{s}: X \times X \rightarrow R^{+}$given by $p^{s}(x, y)=2 p(x, y)-p(x, x)-p(y, y)$ is a metric on $X$.

Each partial metric $p$ on $X$ generates a $T_{0}$ topology $\tau_{p}$ on $X$ which has a base the family of open p-balls $\left\{B_{p}(x, \varepsilon), x \in X, \varepsilon>0\right\}$, where $B_{p}(x, \varepsilon)=\{y \in X: p(x, y)<p(x, x)+\varepsilon\}$ for all $x \in X$ and $\varepsilon>0$.

Definition $2.3(([3]))$ Let $(X, p)$ be a partial metric space.

(i) A sequence $\left\{x_{n}\right\}$ in $(X, p)$ is said to converge to a point $x \in X$ if, and only if $p(x, x)=\lim _{n \rightarrow \infty} p\left(x, x_{n}\right)$.

(ii) A sequence $\left\{x_{n}\right\}$ in $(X, p)$ is said to be Cauchy sequence if the limit: $\lim _{m, n \rightarrow \infty} p\left(x_{n}, x_{m}\right)$ exists and is finite.

(iii) $(X, p)$ is said to be complete if every Cauchy sequence $\left\{x_{n}\right\}$ in $X$ converges, with respect to $\tau_{p}$, to a point $x \in X$, such that

$p(x, x)=\lim _{n, m \rightarrow \infty} p\left(x_{n}, x_{m}\right)$

Lemma $2.4(([3]))$ Let $(X, p)$ be a partial metric space. Then:

(a) $\left\{x_{n}\right\}$ is a Cauchy sequence in $(X, p)$ if, and only if it is a Cauchy sequence in the metric space $\left(X, p^{s}\right)$.

(b) $(X, p)$ is complete if, and only if the metric space $\left(X, p^{s}\right)$ is complete. Furthermore, $\lim _{n \rightarrow \infty} p^{s}\left(x, x_{n}\right)=0$ if, and only if

$p(x, x)=\lim _{n, m \rightarrow \infty} p\left(x_{n}, x\right)=\lim _{n, m \rightarrow \infty} p\left(x_{n}, x_{m}\right)$.

Matthews ([3]) obtained the following Banach fixed point theorem on complete partial metric spaces.

Theorem 2.5 ([3]). Let $f$ be a mapping of a complete partial metric space $(X, p)$ into itself such that there is a real number $c$ with $0 \leq c<1$, satisfying the following condition:

for all $x, y \in X: \quad p(f x, f y) \leq c p(x, y)$,

then $f$ has a fixed point.

In 2010, I. Altun, F.Sola and H. Simsek [1], proved the following result, that generalizes Theorem 1 of Matthews.

Theorem 2.6 [1]. Let $(X, p)$ be a complete partial metric space and let $T: X \rightarrow X$ be a map such that:

$p(T x, T y) \leq \varphi\left(\max \left\{p(x, y), p(x, T x), p(y, T y), \frac{1}{2}[p(x, T y)+p(y, T x)]\right\}\right)$

for all $x, y \in X$, where $\varphi: R^{+} \rightarrow R^{+}$is continuous non-decreasing function such that $\varphi(t)<t$ and the series $\sum_{n \geq 1} \varphi(t)$ converges for all $t 0$. Then $T$ has a unique fixed point.

Very recently, Ljubomir Ciric, B. Samet, H. Aydi and C. Vetro [4], have proved a common fixed point theorem for four mappings satisfying a generalized nonlinear contraction type condition on partial metric spaces and they have given some application related to the homotopy for some operators on a set endowed with a partial metric. The following theorem [4] extended and generalized the results obtained in [1]. 
Theorem 2.7 [4]. Suppose that $A, B, S$ and $T$ are self-maps of a complete partial metric space $(X, p)$ such that $A x \subset T X, B X \subset S X$ and

$p(A x, B y) \leq \varphi(M(x, y))$,

for all $x, y \in X$, where $\varphi \in \Phi$ and

$M(x, y)=\max \left\{p(S x, T y), p(A x, S x), p(B y, T y), \frac{1}{2}[p(S x, B y)+p(A x, T y)]\right\}$

If one of the ranges $A X, B X, T X$ and $S X$ is a closed subset of $(X, p)$, then

(i) A and $S$ have a coincidence point.

(ii) $B$ and $T$ have a coincidence point.

In [5] K.P.R Rao and G.N.V. Kishore have obtained a unique fixed point theorem for self maps satisfying $\psi-\varphi$ contractive condition in partial metric spaces. They generalized and improved some results of Altun et al.[1].

Theorem 2.8 [5]. Let $(X, p)$ be a complete partial metric space and let

$S, T, f, g: X \rightarrow X$

be such that

$\psi(p(S x, T y)) \leq \psi(M(x, y))-\varphi(M(x, y))$ for all $x, y \in X$,

where $\varphi, \psi:[0, \infty[\rightarrow[0, \infty[. \psi$ is continuous, nondecreasing and $\varphi$ is lower semi-continuous with $\varphi(t)<t$ and

$M(x, y)=\max \left\{p\left(f x, g y, p\left(f x, S x, p\left(g y, T y, \frac{1}{2}[p(f x, T y)+p(g y, S x)]\right\}\right.\right.\right.$

(i) $T$ and $F$ have a coincidence point.

(ii) $g$ and $S$ have a coincidence point.

Before stating our main results, we recall the following definitions.

Definition 2.9 Let $X$ be a non-empty set and $T_{1}, T_{2}: X \rightarrow X$ are given self-maps on $X$. The pair $\left(T_{1}, T_{2}\right)$ is said to be weakly compatible if $T_{1} T_{2} t=T_{2} T_{1} t$, whenever $T_{1} t=T_{2} t$ for some $t$ in $X$.

Our main results are the following:

\section{Main Results}

Theorem 3.1 Let $(X, p)$ be a complete partial metric space and let $A, B, S, T: X \rightarrow X$ be such that

$A(X) \subset T(X)$ and $B(X) \subset S(X)$

$\psi(p(A x, B y)) \leq \psi(\theta(x, y))-\varphi(\theta(x, y)) \quad$ for all $x, y \in X$

where

$\theta(x, y)=\lambda p(A x, S x)+\mu p(B y, T y)+\delta p(S x, T y)+\gamma[p(A x, T y)+p(S x, B y)]$

$\mu, \delta, \gamma, \lambda \in] 0,1[$ and $\mu+\delta+2 \gamma+\lambda<1$.

and $\varphi, \psi:[0, \infty[\rightarrow[0, \infty[. \psi$ is continuous, nondecreasing and $\varphi$ is lower semi-continuous, $\varphi(t)=\psi(t)=0 \Longleftrightarrow$ $t=0$. If either $T(X)$ or $S(X)$ is a complete subspace of $X$ and the pairs $(A, S)$ and $(B, T)$ are weakly compatible, then $A, B, S$ and $T$ have a unique common fixed point in $X$. 
Proof. Let $x_{0} \in X$ be any element in $X$. Using (1), we construct sequences $\left(x_{n}\right),\left(y_{n}\right)$ in $X$ such that $\left\{A x_{2 n}=T x_{2 n+1}=y_{2 n} B x_{2 n+1}=S x_{2 n+2}=y_{2 n+1} \quad\right.$ for all $n \geq 1$.

First we prove that, if there exists $n \geq 1$ such that $\theta\left(x_{2 n}, x_{2 n-1}\right)=0$, then $y_{2 n}=y_{2 n-1}$

By taking $x=x_{2 n}$ and $y=x_{2 n-1}$ in (3), we get

$$
\begin{aligned}
0=\theta\left(x_{2 n}, x_{2 n-1}\right)= & \lambda p\left(A x_{2 n}, S x_{2 n}\right)+\mu p\left(B x_{2 n-1}, T x_{2 n-1}\right) \\
& +\delta p\left(S x_{2 n}, T x_{2 n-1}\right) \\
& +\gamma\left[p\left(A x_{2 n}, T x_{2 n-1}\right)+p\left(S x_{2 n}, B x_{2 n-1}\right)\right] \\
= & \lambda p\left(y_{2 n}, y_{2 n-1}\right)+\mu p\left(y_{2 n-1}, y_{2 n-2}\right)+\delta p\left(y_{2 n-1}, y_{2 n-2}\right) \\
& +\gamma\left[p\left(y_{2 n}, y_{2 n-2}\right)+p\left(y_{2 n-1}, y_{2 n-1}\right)\right] .
\end{aligned}
$$

Thus, since $\lambda>0$ and $\lambda p\left(y_{2 n}, y_{2 n-1}\right) \leq \theta\left(x_{2 n}, x_{2 n-1}\right)=0$, it follows that

$p\left(y_{2 n}, y_{2 n-1}\right)=0$,

hence

$y_{2 n}=y_{2 n-1}$

Now we claim if $(7)$ is true, then we have

$y_{2 n}=y_{2 n+1}$,

$$
\left.\begin{array}{rl}
\theta\left(x_{2 n}, x_{2 n+1}\right)= & \lambda p\left(A x_{2 n}, S x_{2 n}\right)+\mu p\left(B x_{2 n+1}, T x_{2 n+1}\right) \\
& +\delta p\left(S x_{2 n}, T x_{2 n+1}\right) \\
& +\gamma\left[p\left(A x_{2 n}, T x_{2 n+1}\right)+p\left(S x_{2 n}, B x_{2 n+1}\right)\right] \\
= & \lambda p\left(y_{2 n}, y_{2 n-1}\right)+\mu p\left(y_{2 n+1}, y_{2 n}\right)+\delta p\left(y_{2 n-1}, y_{2 n}\right) \\
& +\gamma\left[p\left(y_{2 n}, y_{2 n}\right)+p\left(y_{2 n-1}, y_{2 n+1}\right)\right]
\end{array}\right\}
$$

From (9) and by the triangle inequality we get

$$
\begin{aligned}
\theta\left(x_{2 n}, x_{2 n+1}\right) & \leq(\lambda+\delta) p\left(y_{2 n}, y_{2 n+1}\right)+\mu p\left(y_{2 n+1}, y_{2 n}\right) \\
& +\gamma\left[p\left(y_{2 n}, y_{2 n+1}\right)+p\left(y_{2 n}, y_{2 n-1}\right)-p\left(y_{2 n}, y_{2 n}\right)+p\left(y_{2 n}, y_{2 n}\right)\right]
\end{aligned}
$$

Hence

$\theta\left(x_{2 n}, x_{2 n+1}\right) \leq(\gamma+\delta+\lambda) p\left(y_{2 n-1}, y_{2 n}\right)+(\mu+\gamma) p\left(y_{2 n}, y_{2 n+1}\right)$.

Since

$p\left(y_{2 n}, y_{2 n-1}\right)=p\left(y_{2 n}, y_{2 n}\right) \leq p\left(y_{2 n}, y_{2 n+1}\right)$,

then from (10), (11) and (4) we obtain

$$
\left.\begin{array}{rl}
\theta\left(x_{2 n}, x_{2 n+1}\right) & \leq(\lambda+\mu+\delta+2 \gamma) p\left(y_{2 n}, y_{2 n+1}\right) \\
& <p\left(y_{2 n}, y_{2 n+1}\right)
\end{array}\right\}
$$

Since $\psi$ is monotone, then

$\psi\left(\theta\left(x_{2 n}, x_{2 n+1}\right)\right) \leq \psi\left(p\left(y_{2 n}, y_{2 n+1}\right)\right)$,

$p(A x, B y)=p\left(A x_{2 n}, B x_{2 n+1}\right)=p\left(y_{2 n}, y_{2 n+1}\right)$. 
From $(13),(14)$ and (2) we get

$\psi\left(p\left(y_{2 n}, y_{2 n+1}\right)\right) \leq \psi\left(p\left(y_{2 n}, y_{2 n+1}\right)\right)-\varphi\left(\theta\left(x_{2 n}, x_{2 n+1}\right)\right)$.

By the property of $\varphi$, we have $\varphi\left(\theta\left(x_{2 n}, x_{2 n+1}\right)\right)=0$, this implies that

$\theta\left(x_{2 n}, x_{2 n+1}\right)=0$.

By the fact that $\lambda>0$ and $\lambda p\left(y_{2 n}, y_{2 n+1}\right) \leq \theta\left(x_{2 n}, x_{2 n+1}\right)=0$, therefore $y_{2 n}=y_{2 n+1}$. Continuing in this way, we can conclude that $y_{n}=y_{n+k}$ for all $k \geq 0$. Thus, the sequence $\left\{y_{n}\right\}$ is a Cauchy sequence. Now we can suppose that

$\theta\left(x_{2 n}, x_{2 n+1}\right)=0$ for all $n \geq 1$.

Setting $p_{2 n}=p\left(y_{2 n}, y_{2 n+1}\right)$. We claim that

$p_{2 n+1} \leq p_{2 n}$ for all $n \geq 1$

Suppose (16) is not true, that is, there exists $n \in N$ such that $p_{2 n+1}>p_{2 n}$, then

$$
\begin{aligned}
& \psi\left(p_{2 n}\right) \leq \psi\left(p_{2 n+1}\right)=\left(p\left(y_{2 n+1}, y_{2 n+2}\right)\right)=\psi\left(p\left(A x_{2 n+1}, B x_{2 n+2}\right)\right) \\
& \leq \psi\left(\theta\left(x_{2 n+1}, x_{2 n+2}\right)\right)-\varphi\left(\theta\left(x_{2 n+1}, x_{2 n+2}\right)\right) \\
& \theta\left(x_{2 n+2}, x_{2 n+1}\right)= \lambda p\left(A x_{2 n+2}, S x_{2 n+2}\right)+\mu p\left(B x_{2 n+1}, T x_{2 n+1}\right) \\
&+\delta p\left(S x_{2 n+2}, T x_{2 n+1}\right) \\
&+\gamma\left[p\left(A x_{2 n+2}, T x_{2 n+1}\right)+p\left(S x_{2 n+2}, B x_{2 n+1}\right)\right] \\
&= \lambda p\left(y_{2 n+2}, y_{2 n+1}\right)+\mu p\left(y_{2 n+1}, y_{2 n}\right)+\delta p\left(y_{2 n+1}, y_{2 n}\right) \\
&+\gamma\left[p\left(y_{2 n+2}, y_{2 n}\right)+p\left(y_{2 n+1}, y_{2 n+1}\right)\right] .
\end{aligned}
$$

Then by triangle inequality, we get

$\theta\left(x_{2 n+2}, x_{2 n+1}\right) \leq \lambda p_{2 n+1}+\mu p_{2 n}+\delta p_{2 n+1}+\gamma p_{2 n+1}+\gamma p_{2 n}$.

Since

$p\left(y_{2 n+1}, y_{2 n+2}\right) \leq p\left(y_{2 n}, y_{2 n+1}\right)$

$\lambda+\mu+\delta+2 \gamma<1$, then from (16) we have

$\theta\left(x_{2 n+1}, x_{2 n+2}\right) \leq(\lambda+\mu+\delta+2 \gamma) p_{2 n}$

$$
\leq p_{2 n}
$$

$\psi$ is monotone, we have

$\psi\left(p_{2 n}\right) \leq \psi\left(p_{2 n}\right)-\varphi\left(\theta\left(x_{2 n+1}, x_{2 n+2}\right)\right)$.

This implies $\varphi\left(\theta\left(x_{2 n}, x_{2 n+1}\right)\right)=0$, by the property of $\varphi$, it follows that $\theta\left(x_{2 n}, x_{2 n+1}\right)=0$, which is a contradiction with (15). With the same way, we prove

$p_{2 n+2} \leq p_{2 n+1}$ for all $n \geq 1$

Thus from (16) and from (17) we have

$p_{n+1} \leq p_{n}$ for all $n \geq 1$

Hence, the sequence $\left\{p_{n}\right\}$ is a non-decreasing sequence of non negative real numbers and must convergence to a real number denoted by $l$. Say:

$\lim _{n \rightarrow \infty} p\left(y_{n}, y_{n+1}\right)=l, \quad l \geq 0$ 
We shall prove that $l=0$. We suppose that

$l>0$,

then from (9) and (12) we get

$$
\begin{aligned}
\lim _{n \rightarrow \infty} \lambda p\left(A x_{2 n}, S x_{2 n}\right) & =\lim _{n \rightarrow \infty} \lambda p\left(y_{2 n}, y_{2 n-1}\right) \leq \limsup _{n \rightarrow \infty} \theta\left(x_{2 n}, x_{2 n+1}\right) \\
& \leq(\lambda+\mu+\delta+2 \gamma) \lim _{n \rightarrow \infty} p\left(y_{2 n}, y_{2 n+1}\right),
\end{aligned}
$$

this implies, by using (4), that

$0<\lambda l \leq \limsup _{n \rightarrow \infty} \theta\left(x_{2 n}, x_{2 n+1}\right) \leq l$,

so, there exists $l_{1}>0$ and a subsequence $\left\{x_{2 n_{k}}\right\}$ of $\left\{x_{2 n}\right\}$ such that

$\lim _{n \rightarrow \infty} \theta\left(x_{2 n_{k}}, x_{2 n_{k}+1}\right)=l_{1} \leq l$.

Hence, by the lower semicontinuity of $\varphi$, we have

$\varphi\left(l_{1}\right) \leq \liminf _{k \rightarrow \infty} \varphi\left(\theta\left(x_{2 n_{k}}, x_{2 n_{k}+1}\right)\right)$

From (2), we get

$\psi\left(p\left(y_{2 n_{k}}, y_{2 n_{k}+1}\right)\right) \leq \psi\left(\theta\left(x_{2 n_{k}}, x_{2 n_{k}+1}\right)\right)-\varphi\left(\theta\left(x_{2 n_{k}}, x_{2 n_{k}+1}\right)\right)$

Taking the upper limit as $k \rightarrow \infty$ in (20), we obtain

$$
\begin{aligned}
\psi(l) & \leq \psi\left(l_{1}\right)-\liminf _{k \rightarrow \infty} \varphi\left(\theta\left(x_{2 n_{k}}, x_{2 n_{k}+1}\right)\right) \\
& \leq \psi\left(l_{1}\right)-\varphi\left(l_{1}\right) \\
& \leq \psi(l)-\varphi\left(l_{1}\right) .
\end{aligned}
$$

This implies that $\varphi\left(l_{1}\right)=0$. Thus, by the property of $\varphi$, we have $l_{1}=0$, which is a contradiction with $(18)$. Therefore $l=0$ and so

$\lim _{n \rightarrow \infty} p\left(y_{n}, y_{n+1}\right)=0$,

and from $\left(p_{2}\right)$, we have also

$\lim _{n \rightarrow \infty} p\left(y_{n}, y_{n}\right)=0$,

from (21) and (22), we have

$\lim _{n \rightarrow \infty} p^{s}\left(y_{n}, y_{n+1}\right)=0$.

Now, we prove that $\left\{y_{2 n}\right\}$ is a Cauchy sequence in $\left(X, p^{s}\right)$. On contrary, suppose that $\left\{y_{2 n}\right\}$ is not a Cauchy sequence in $\left(X, p^{s}\right)$. There exists an $\varepsilon>0$ and monotone increasing sequences of natural numbers $\left\{2 m_{k}\right\}$ and $\left\{2 n_{k}\right\}$ such that $n_{k}<m_{k}$ and

$p^{s}\left(y_{2 m_{k}}, y_{2 n_{k}}\right) \geq \varepsilon$

and

$p^{s}\left(y_{2 m_{k}}, y_{2 n_{k}-2}\right)<\varepsilon$

From $(24)$ and $(25)$ we get

$$
\begin{aligned}
\varepsilon & \leq p^{s}\left(y_{2 m_{k}}, y_{2 n_{k}}\right) \\
& \leq p^{s}\left(y_{2 m_{k}}, y_{2 n_{k}-2}\right)+p^{s}\left(y_{2 n_{k}-2}, y_{2 n_{k}-1}\right)+p^{s}\left(y_{2 n_{k}-1}, y_{2 n_{k}}\right) \\
& <\varepsilon+p^{s}\left(y_{2 n_{k}-2}, y_{2 n_{k}-1}\right)+p^{s}\left(y_{2 n_{k}-1}, y_{2 n_{k}}\right) .
\end{aligned}
$$


Letting $k \rightarrow \infty$ and using (23), we have

$\lim _{k \rightarrow \infty} p^{s}\left(y_{2 m_{k}}, y_{2 n_{k}}\right)=\varepsilon$.

Hence from the definition of $p^{s}$ and from (22), we have

$\lim _{k \rightarrow \infty} p\left(y_{2 m_{k}}, y_{2 n_{k}}\right)=\frac{\varepsilon}{2}$.

Letting $k \rightarrow \infty$ and using (26), (24) in

$\left|p^{s}\left(y_{2_{m_{k}}}, y_{2 n_{k}+1}\right)-p^{s}\left(y_{2_{m_{k}}}, y_{2 n_{k}}\right)\right| \leq p^{s}\left(y_{2_{n_{k}}+1}, y_{2 n_{k}}\right)$,

we obtain

$\lim _{k \rightarrow \infty} p^{s}\left(y_{2 m_{k}}, y_{2 n_{k}+1}\right)=\varepsilon$.

Hence, we have

$\lim _{k \rightarrow \infty} p\left(y_{2 m_{k}}, y_{2 n_{k}+1}\right)=\frac{\varepsilon}{2}$.

Letting $k \rightarrow \infty$ and using (26), (24) in

$\left|p^{s}\left(y_{2 m_{k}-1}, y_{2 n_{k}+1}\right)-p^{s}\left(y_{2 m_{k}}, y_{2 n_{k}}\right)\right| \leq p^{s}\left(y_{2 m_{k}-1}, y_{2 m_{k}}\right)$,

we get:

$\lim _{k \rightarrow \infty} p^{s}\left(y_{2 m_{k}-1}, y_{2 n_{k}}\right)=\varepsilon$.

Hence, we have

$\lim _{k \rightarrow \infty} p\left(y_{2 m_{k}-1}, y_{2 n_{k}}\right)=\frac{\varepsilon}{2}$.

Letting $k \rightarrow \infty$ and using (30), (24) in

$\left|p^{S}\left(y_{2 m_{k}-1}, y_{2 n_{k}+1}\right)-p^{S}\left(y_{2 m_{k}-1}, y_{2 n_{k}}\right)\right| \leq p^{S}\left(y_{2 n_{k}+1}, y_{2 n_{k}}\right)$.

we get:

$\lim _{k \rightarrow \infty} p^{S}\left(y_{2 m_{k}-1}, y_{2 n_{k}+1}\right)=\varepsilon$.

Hence, we have

$\lim _{k \rightarrow \infty} p\left(y_{2 m_{k}-1}, y_{2 n_{k}+1}\right)=\frac{\varepsilon}{2}$.

Now, by (2) and (3) we have

$$
\left.\begin{array}{rl}
\psi\left(p\left(A x_{2 m_{k}}, B x_{2 n_{k}+1}\right)\right)=\psi( & \left.\left(y_{2 m_{k}}, y_{2 n_{k}+1}\right)\right) \\
\leq \psi & \left.\theta\left(x_{2 m_{k}}, x_{2 n_{k}+1}\right)\right)-\varphi\left(\theta\left(x_{2 m_{k}}, x_{2 n_{k}+1}\right)\right), \\
\left.\qquad x_{2 m_{k}}, x_{2 n_{k}+1}\right)= & \lambda p\left(y_{2 m_{k}}, y_{2 m_{k}-1}\right)+\mu p\left(y_{2 n_{k}+1}, y_{2 n_{k}}\right) \\
& +\delta p\left(y_{2 m_{k}-1}, y_{2 n_{k}}\right) \\
& +\gamma\left[p\left(y_{2 m_{k}}, y_{2 n_{k}}\right)+p\left(y_{2 m_{k}-1}, y_{2 n_{k+1}}\right)\right]
\end{array}\right\}
$$

Letting $k \rightarrow \infty$ and using (21), (27), (31), (34) and since $\gamma+\frac{\delta}{2} \leq \frac{1}{2}$, we obtain

$\begin{aligned} 0.3 \mathrm{~cm} \psi\left(\frac{\varepsilon}{2}\right) & \leq \psi\left(\left(\gamma+\frac{\delta}{2}\right) \varepsilon\right)-\varphi\left(\left(\gamma+\frac{\delta}{2}\right) \varepsilon\right) \\ & \leq \psi\left(\frac{\varepsilon}{2}\right)-\varphi\left(\left(\gamma+\frac{\delta}{2}\right) \varepsilon\right)\end{aligned}$ 
this implies that $\varphi\left(\left(\gamma+\frac{\delta}{2}\right) \varepsilon\right)=0$, then $\varepsilon=0$; which is a contradiction. Hence $\left\{y_{2 n}\right\}$ is a Cauchy sequence. Letting $n \rightarrow \infty$ and $m \rightarrow \infty$ in

$\left|p^{s}\left(y_{2 n+1}, y_{2 m+1}\right)-p^{s}\left(y_{2 m}, y_{2 n}\right)\right| \leq p^{s}\left(y_{2 n+1}, y_{2 n}\right)+p^{s}\left(y_{2 m+1}, y_{2 m}\right)$,

we get $\lim _{n . m \rightarrow \infty} p^{s}\left(y_{2 n+1}, y_{2 m+1}\right)=0$. Hence $\left\{y_{2 n+1}\right\}$ is a Cauchy sequence. Thus $\left\{y_{n}\right\}$ is a Cauchy sequence in $\left(X, p^{s}\right)$. We have $\lim _{n . m \rightarrow \infty} p^{s}\left(y_{n}, y_{m}\right)=0$. Now, from the definition of $p^{s}$ and from (22), we have

$\lim _{n . m \rightarrow \infty} p\left(y_{n}, y_{m}\right)=0$.

Suppose $S(X)$ is complete. Since $\left\{y_{2 n+1}\right\} \subset S(X)$ is a Cauchy sequence in the complete metric space $\left(S(X), p^{s}\right)$, therefore there exists $t \in X$ such that $v=S(t) \in S(X)$. Since $\left\{y_{n}\right\}$ is a Cauchy sequence in $\left(X, p^{s}\right)$ and $y_{2 n+1} \rightarrow v$, it follows that $y_{2 n} \rightarrow v$. From Lemma 1 (b), we have

$p(v, v)=\lim _{n \rightarrow \infty} p\left(y_{2 n+1}, v\right)=\lim _{n \rightarrow \infty} p\left(y_{2 n}, v\right)=\lim _{n . m \rightarrow \infty} p\left(y_{n}, y_{m}\right)$

From (35) and (36), we have

$p(v, v)=\lim _{n \rightarrow \infty} p\left(y_{2 n+1}, v\right)=\lim _{n \rightarrow \infty} p\left(y_{2 n}, v\right)=0$

We shall prove that $\lim _{n \rightarrow \infty} p\left(A t, y_{2 n}\right)=p(A t, v)$. Letting $n \rightarrow \infty$ in

$p^{s}\left(A t, y_{2 n}\right)=2 p\left(A t, y_{2 n}\right)-p(A t, A t)-p\left(y_{2 n}, y_{2 n}\right)$

we get by using (22)

$p^{s}(A t, v)=2 \lim _{n \rightarrow \infty} p\left(A t, y_{2 n}\right)-p(A t, A t)-0$

$2 p(A t, v)-p(A t, A t)-p(v, v)=2 \lim _{n \rightarrow \infty} p\left(A t, y_{2 n}\right)-p(A t, A t)$

By (37), we have

$p(A t, v)=\lim _{n \rightarrow \infty} p\left(A t, y_{2 n}\right)$

Let $A t \neq v$

$p(A t, v) \leq p\left(A t, B x_{2 n+1}\right)+p\left(B x_{2 n+1}, v\right)-p\left(B x_{2 n+1}, B x_{2 n+1}\right)$

$\leq p\left(A t, B x_{2 n+1}\right)+p\left(y_{2 n+1}, v\right)$

$\psi(p(A t, v)) \leq \psi\left(p\left(A t, B x_{2 n+1}\right)+p\left(y_{2 n+1}, v\right)\right)$

Hence letting $n \rightarrow \infty$ in (38), we obtain

$$
\begin{aligned}
\psi(p(A t, v)) & \leq \psi\left(\lim _{n \rightarrow \infty} p\left(A t, B x_{2 n+1}\right)+0\right) \\
& =\lim _{n \rightarrow \infty} \psi\left(p\left(A t, B x_{2 n+1}\right)\right) \\
\leq & \lim _{n \rightarrow \infty}\left[\psi\left(\theta\left(t, x_{2 n+1}\right)\right)-\varphi\left(\theta\left(t, x_{2 n+1}\right)\right)\right] \\
\theta\left(\left(t, x_{2 n+1}\right)\right) & =\lambda p\left(A t, S x_{2 n+1}\right)+\mu p\left(B x_{2 n+1}, T x_{2 n+1}\right) \\
& +\delta p\left(S t, T x_{2 n+1}\right)+\gamma\left[p\left(A t, T x_{2 n+1}\right)+p\left(S t, B x_{2 n+1}\right)\right]
\end{aligned}
$$

Then

$$
\begin{aligned}
\theta\left(\left(t, x_{2 n+1}\right)\right) & =\lambda p\left(A t, y_{2 n}\right)+\mu p\left(y_{2 n+1}, y_{2 n}\right) \\
& +\delta p\left(v, y_{2 n}\right)+\gamma\left[p\left(A t, y_{2 n}\right)+p\left(v, y_{2 n+1}\right)\right]
\end{aligned}
$$


Letting $n \rightarrow \infty$ in (40) and using (21), (37) and the fact that $\lambda+\gamma<1$, we obtain

$\lim _{n \rightarrow \infty} \theta\left(\left(t, x_{2 n+1}\right)\right)=(\lambda+\gamma) p(v, A t) \leq p(v, A t)$

Thus

$\psi(p(A t, v)) \leq \psi(p(A t, v))-\varphi((\lambda+\gamma) p(v, A t))$

It follows $\varphi((\lambda+\gamma) p(v, A t))=0$, from the property of $\varphi$ we have $p(v, A t)=0$ hence $v=A t=S t$. Since the pair $(A, S)$ are compatible, We have $A v=S v$. Suppose

$S v \neq v$

As in above, using the metric $p^{s}$ and (22), (37), we can show that

$$
\begin{aligned}
p(A v, v) & =\lim _{n \rightarrow \infty} p\left(A v, y_{2 n}\right) \\
p(A v, v) & \leq\left[p\left(A v, B x_{2 n+1}\right)+p\left(B x_{2 n+1}, v\right)-p\left(B x_{2 n+1}, B x_{2 n+1}\right)\right] \\
& \leq p\left(A v, B x_{2 n+1}\right)+p\left(y_{2 n+1}, v\right)
\end{aligned}
$$

Then

$\psi(p(A v, v)) \leq \psi\left(p\left(A v, B x_{2 n+1}\right)+p\left(y_{2 n+1}, v\right)\right)$

Letting $n \rightarrow \infty$, we get

$$
\begin{aligned}
\psi(p(A v, v)) & \leq \psi\left(\lim _{n \rightarrow \infty} p\left(A v, B x_{2 n+1}\right)\right)+0 \\
& =\lim _{n \rightarrow \infty} \psi\left(p\left(A v, B x_{2 n+1}\right)\right) \\
& \leq \lim _{n \rightarrow \infty}\left[\psi\left(\theta\left(v, x_{2 n+1}\right)\right)-\varphi\left(\theta\left(v, x_{2 n+1}\right)\right)\right]
\end{aligned}
$$

Since

$$
\begin{gathered}
\theta\left(v, x_{2 n+1}\right)=\lambda p(A v, A v)+\mu p\left(y_{2 n}, y_{2 n+1}\right)+\delta p\left(A v, y_{2 n}\right) \\
+\gamma\left[p\left(y_{2 n}, A v\right)+p\left(A v, y_{2 n+1}\right)\right] \\
\lim _{n \rightarrow \infty} \theta\left(v, x_{2 n+1}\right)=\lambda p(A v, A v,)+0+\delta p(A v, v) \\
+2 \gamma p(A v, v)
\end{gathered}
$$

Since $\lambda+\delta+2 \gamma<1$, We obtain

$\lim _{n \rightarrow \infty} \theta\left(v, x_{2 n+1}\right)=(\lambda+\delta+2 \gamma) p(A v, v) \leq p(A v, v)$

Thus

$\psi(p(A v, v)) \leq \psi(p(A v, v))-\varphi((\lambda+\mu+2 \gamma) p(A v, v))$

Hence $\varphi((\lambda+\mu+2 \gamma) p(A v, v))=0$, by the property of $\varphi$, we have

$A v=v=S v$

Since $A(X) \subset T(X)$, there exists $w \in X$ such that $v=S v=T w$. Suppose $v \neq B w$

$$
\begin{aligned}
\psi(p(v, B w))= & \psi(p(A v, B w)) \leq \psi(\theta(v, w))-\varphi(\theta(v, w)) \\
\theta(v, w)= & \lambda p(A v, S v)+\mu p(B w, T w)+\delta p(S v, T w) \\
& +\gamma[p(A v, T w)+p(S v, B w)] \\
= & \lambda p(v, v)+\mu p(B w, v)+\delta p(v, v) \\
& +\gamma[p(v, v)+p(v, B w)] \\
= & (\lambda+\delta+\gamma) p(v, v)+(\mu+\gamma) p(v, B w) \\
= & 0+(\mu+\gamma) p(v, B w) \\
\leq & p(v, B w) .
\end{aligned}
$$


Hence

$\psi(p(v, B w)) \leq \psi(p(v, B w))-\varphi((\mu+\gamma) p(v, B w))$

Thus, $\varphi((\mu+\gamma) p(v, B w))=0$. By the property of $\varphi$, we have $v=B w$. Thus $T w=B w=v$. Since $(T, B)$ is weakly compatible, we have $T v=B v$. Suppose $B v \neq v$.

$$
\begin{aligned}
\psi(p(v, B v))= & \psi(p(A v, B v)) \leq \psi(\theta(v, v))-\varphi(\theta(v, v)) \\
\theta(v, v)= & \lambda p(A v, S v)+\mu p(B v, T v)+\delta p(S v, T v) \\
& +\gamma[p(A v, T v)+p(S v, B v)] \\
= & \lambda p(v, v)+\mu p(B v, B v)+\delta p(v, B v) \\
& +\gamma[p(v, B v)+p(v, B v)] \\
= & \mu p(B v, B v)+(\delta+2 \gamma) p(v, B v) \quad\left(\text { Fromp }_{2}\right) \\
= & (\mu+\delta+2 \gamma) p(v, B v) \\
\leq & p(v, B v)
\end{aligned}
$$

hence

$\psi(p(v, B v)) \leq \psi(p(v, B v))-\varphi((\mu+\delta+2 \gamma) p(v, B v))$

It follows $\varphi((\mu+\delta+2 \gamma) p(v, B v))=0$, then from the property of $\varphi$, we have $p(v, B v)=0$, thus $v=B v$. We have.

$T v=B v=v$.

From (42) and (43), $v$ is a common fixed point of $A, B, T$ and $S$. Now we prove the uniqueness of the common fixed point. Let $z$ be another common fixed point of $A, B, T$ and $S$. Suppose $v \neq z$,

$\psi(p(v, z))=\psi(p(A v, B z)) \leq \psi(\theta(v, z))-\varphi(\theta(v, z))$,

$$
\begin{aligned}
\theta(v, z)= & \lambda p(A v, S v)+\mu p(B z, T z)+\delta p(S v, T z) \\
& +\gamma[p(A v, T z)+p(S v, B z)] \\
= & \lambda p(v, v)+\mu p(z, z)+\delta p(v, z) \\
& +\gamma[p(v, z)+p(v, z)] \\
= & (\delta+\mu+2 \gamma) p(v, z) \text { From } p_{2} \\
\leq & p(v, z)
\end{aligned}
$$

Hence

$\psi(p(v, z)) \leq \psi(p(v, z))-\varphi((\delta+\mu+2 \gamma) p(v, z))$

It follows that $\varphi((\delta+\mu+2 \gamma) p(v, z))=0$ and by the property of $\varphi$, we have $v=z$. Thus $v$ is the unique common fixed point of $A, B, T$ and $S$.

Corollary 3.2 Let $(X, p)$ be a partial metric space and let $A, B, S, T: X \rightarrow X$ be such that $A(X) \subset T(X)$ and $B(X) \subset S(X)$

If

$p(A x, B x) \leq \theta(x, y)$ for all $x, y \in X$,

where

$$
\begin{aligned}
\theta(x, y)= & \lambda p(A x, S x)+\mu p(B y, T y)+\delta p(S x, T y) \\
& +\gamma[p(A x, T y)+p(S x, B y)]
\end{aligned}
$$

$\mu, \delta, \gamma, \lambda \in[0.1[\quad$ and $\quad \lambda 0, \mu+\delta+2 \gamma+\lambda<1$,

if either $T(X)$ or $S(X)$ is a complete subspace of $X$ and the pairs $(A, S)$ and $(B, T)$ are weakly compatible, then $A, B, S$ and $T$ have a unique common fixed point in $X$. 
Proof. Taking $\psi(t)=t$ and $\varphi=0$ in theorem 3.1 .

Corollary 3.3 Let $(X, p)$ be a partial metric space and let $A, T: X \rightarrow X$ be such that

$A(X) \subset T(X)$.

If

$p(A x, A x) \leq \theta(x, y)$ for all $x, y \in X$,

where

$$
\begin{aligned}
\theta(x, y)= & \lambda p(A x, T x)+\mu p(A y, T y)+\delta p(T x, T y) \\
& +\gamma[p(A x, T y)+p(T x, A y)]
\end{aligned}
$$

$\mu, \delta, \gamma, \lambda \in[0.1[$ and $\lambda 0, \mu+\delta+2 \gamma+\lambda<1$,

if $T(X)$ is a complete subspace of $X$ and the pairs $(A, T)$ are weakly compatible, then $A$ and $T$ have a unique common fixed point in $X$.

Proof. Taking $\psi(t)=t$ and $\varphi=0$ and $A=B$ and $T=S$ in theorem 5.

Example 3.4 Let $X=[0,1]$ and $p(x, y)=\max \{x, y\}$ for $x, y \in X$. Let $A, B, S$ and $T: X \rightarrow X$ and

- $S(x)=\frac{x}{2}, T(x)=\frac{x}{3}, A(x)=\frac{x}{4}, B(x)=\frac{x}{6}$,

- $\psi:[0, \infty[\rightarrow[0, \infty[$ defined by: $\psi(t)=t$,

- $\varphi:\left[0, \infty\left[\rightarrow\left[0, \infty\left[\right.\right.\right.\right.$ defind by $\varphi(t)=\frac{t}{2}$,

- $\lambda=\beta=\gamma=\delta=\frac{1}{6}$.

Then all conditions of theorem 3.1 are satisfied and 0 is the unique fixed point of $A, B, S$ and $T$.

Example 3.5 Let $X=[0,1]$ and $p(x, y)=\max \{x, y\}$ for $x, y \in X$. Let $A, B, S$ and $T: X \rightarrow X$ and

- $S(x)=\frac{x}{x+1}, T(x)=\frac{x}{x+2}, \quad A(x)=\frac{x^{2}}{2 x+2} \quad$ and $B(x)=\frac{x^{2}}{2 x+4}$, $\psi:[0, \infty[\rightarrow[0, \infty[$ defined by $\psi(t)=t$, $\varphi:\left[0, \infty\left[\rightarrow\left[0, \infty\left[\right.\right.\right.\right.$ by $\varphi(t)=\frac{t}{2}$,

- $\lambda=\beta=\gamma=\delta=\frac{1}{6}$.

Then all conditions of theorem 3.1 are satisfied and 0 is the unique fixed point of $A, B, S$ and $T$.

\section{Applications}

In this section, we give an application of the previous section.

Set $Y=\{\chi:[0, \infty[\rightarrow[0, \infty[, \quad \chi$ is a Lebesgue integrable mapping which is summable and nonegative and satisfies $\int_{0}^{\varepsilon} \chi(t) \mathrm{dt}>0$ for each $\left.\varepsilon>0\right\}$.

Theorem 4.1 Let $(X, p)$ be a complete partial metric space and let $A, B, S, T: X \rightarrow X$ be such that

$A(X) \subset T(X)$ and $B(X) \subset S(X)$

and for all $x, y \in X$ :

$\int_{0}^{\psi(p(A x, B y))} \chi(t) d t \leq \int_{0}^{\psi(\theta(x, y))} \chi(t) d t-\int_{0}^{\varphi(\theta(x, y))} \chi(t) d t, \quad \chi \in Y$,

where

$$
\left.\begin{array}{c}
\theta(x, y)=\begin{array}{c}
\lambda p(A x, S x)+\mu p(B y, T y)+\delta p(S x, T y) \\
+\gamma[p(A x, T y)+p(S x, B y)] \\
0.2 c m
\end{array} \\
\mu, \delta, \gamma, \lambda \in] 0.1[\text { and } \mu+\delta+2 \gamma+\lambda<1
\end{array}\right\}
$$

and $\varphi, \psi:[0, \infty[\rightarrow[0, \infty[. \psi$ is continuous, nondecreasing and $\varphi$ is lower semi-continuous, $\varphi(t)=\psi(t)=0 \Longleftrightarrow$ $t=0$. If either $T(X)$ or $S(X)$ is a complete subspace of $X$ and the pairs $(A, S)$ and $(B, T)$ are weakly compatible, then $A, B, S$ and $T$ have a unique common fixed point in $X$. 
Proof. Define $\Lambda: R_{+} \rightarrow R_{+}$by $\Lambda(x)=\int_{0}^{x} \chi(t) d t$. then $\Lambda$ is continuous and nondecreasing with $\Lambda(0)=0$. Then we obtain

$\Lambda(\psi(p(A x, B y)) \leq \psi(p(A x, B y))-\varphi(p(A x, B y))$

Which further can be written as

$\Psi_{1}\left(p(A x, B y) \leq \Psi_{1}(\theta(x, y))-\Phi_{1}(\theta(x, y))\right.$

where $\Psi_{1}=\Lambda \circ \psi$ and $\Phi_{1}=\Lambda \circ \varphi$. Hence by theorem 3.1 we have the desired results.

\section{References}

[1] Altun.F. Sola, H. Simse: Generalized contractions on partial metric spaces, Topology and Applications. 157, (2010), 6, 2778-2785.

[2] I. Altun and H.K. Nashine: Fixed Point Theorems for generalized weakly contractive condition in ordered metric spaces. hindawi publishing corporation, fixed point theory and Applications Vol. 2011, article ID 132367,20 pages doi 10. $1155 / 2011 / 132367$

[3] S.G.Matthews, Partial metric topology, in : Proc. 8th Summer Conference on General Topology and Applications, in: Ann. New York Acad. Sci. Vol. 728, 1994, pp. 183-197

[4] Lj. Ciric, B. Samet, H. Aydi, C. Vetro: Common fixed points of generalized contractions on partial metric spaces and an application. Appl. Maths. comp. 218(2011) pp. 2398-2406.

[5] K.P.R. Rao and GNV. Kishore: A unique common fixed point theorem for four maps under $\varphi-\psi$ contractive condition in Partial metric spaces. Bul. of Math. Analy. and Appl. ISSN 1821-1291. Vol. 3 (2011), pp. 56-63.

[6] R. Kopperman, S.G.Matthews and H. Pajoohesh. What do partial metrics represent? Spatial representation: discret vs. continuous computational models. dagstuhl Seminar Proceeding, n0. 04351, Internationales Begegnungs-und Forschungszentrum fuer Informatik (IBFI), Schloss Dagstuhl, Germany (2005). MR 2005j: 54007.

[7] S.J. Oneill: partial metrics, valuations and domain theory, im Proc. 11th. Summer Conference on General Topology and Applications. Vol. 806 of Annals of the New-york Academy partial metrics valuations and domain theory of Sciemces, 1996, pp. 304-315, The New-York Academy of sciences, New-York, NY, USA.

[8] S. Oltra:, O. Valero: Banachs fixed point theorem for Partial metric spaces. Rend. Istit. Mat. Univ. Triest. Vol. XXXVI, 2004, pp. 17-26.

[9] S. Romaguera: A kirk type characterization of completeness for partial metric spaces. fixed point theory. Vol. 2010. Article Id 493298, 6 pages, doi. 10. 1155/2010/493298. 